\title{
Enhanced FTIR Spectroscopy of Biological Liquid Samples Confined Between Ge Hemispherical ATR Element and Al Coated Glass Substrate
}

\author{
Alex Gunko ${ }^{1}$, Yurii K Gun'ko ${ }^{2}$ and Tatiana S Perova ${ }^{3 *}$ \\ ${ }^{1}$ Cork University Hospital, Wilton, Cork, Ireland \\ ${ }^{2}$ School of Chemistry, Trinity College Dublin, The University of Dublin, Ireland \\ ${ }^{3}$ Department of Electronic and Electrical Engineering, Trinity College Dublin, The University of Dublin, Ireland \\ *Corresponding author: Tatiana S Perova, Department of Electronic and Electrical Engineering, Trinity College Dublin, The \\ University of Dublin, Ireland
}

\section{ARTICLE INFO}

Received: 幽 December 30, 2019

Published: 幽January 08, 2020

Citation: Alex Gunko, Yurii K Gun'ko, Tatiana S Perova. Enhanced FTIR Spectroscopy of Biological Liquid Samples Confined Between Ge Hemispherical ATR Element and $\mathrm{Al}$ Coated Glass Substrate. Biomed J Sci \& Tech Res 24(3)-2020. BJSTR. MS.ID.004049.

\section{ABSTRACT}

We demonstrated a modified SEIRA approach, which can be easily utilized for measurements of various liquid samples, including biological specimens. In addition, the possibility of using Al, known as non-typical SERS metal, for enhancement of IR signal was demonstrated with enhancement factor varied from 10 to 1000 for different IR bands. This method is easily accessible and does not require deposition of metal on expensive internal reflection element. Instead metal can be deposited on cheap glass substrate, which is used in the suggested set up as top window during SEIRA measurements. Finally, the thickness of the analyte can be controlled by the position of the pressure applicator when using a grazing angle ATR attachment.

Abbreviations : IR: Infrared; FTIR: Fourier Transform Infrared; ATR: Attenuated Total Reflection; IRRAS: IR Reflection Absorption Spectroscopy; SERS: Surface Enhanced Raman Spectroscopy; BSA: Bovine Serum Albumin; GATR: Grazing angle Attenuated Total Reflection; IRE: Internal Reflection Element; EM: Electromagnetic; EMA: Effective-Medium Approximation; SEIRA: Surface Enhanced IR Absorption

\section{Introduction}

It is well known that Infrared (IR) spectroscopy is a widely used as an effective analytical technique for investigation of molecular structure and composition as well as intermolecular interactions of specific and non-specific nature in various substances. However, this technique has some limitations in particular when applied to minute trace concentration of substances as well as for the aqueous solution of biological species and for single molecule detection. Over last few decades different methods of Fourier Transform Infrared (FTIR) spectroscopy were developed to increase the sensitivity of this technique specifically for characterization of different biological samples (including proteins, DNA and RNA, cells and tissue specimens, etc.) as well as a single molecule detection [1-5]. Laboratory analysis of biological fluids is of great importance for medical diagnostics and health science in general. Mid-infrared spectroscopy, using the Attenuated Total Reflection (ATR) technique, showed great potential for the quantitative multicomponent analysis of various body fluids [5-9]. There are many benefits in using Mid-infrared spectroscopy coupled to ATR including: trace samples can be measured; minimal sample preparation is required; no additional reagents or disposables are needed; continuous measurements and bulk measurements are easy to carry out; the concentrations of several analytes can be measured simultaneously from a single spectrum.

Recently developed new FTIR methods involve various modifications of the Attenuated Total Reflection (ATR) techniques, including single and multiple reflections approach [1,2]. Different 
optical internal reflection elements "(named ATR or IRE), such as hemispherical prism and trapezoidal elements, made from materials with high refractive index were developed for these methods. This resulted in significant enhancements of the infrared signal due to the grazing angle incidence (and reflection) and multiple reflections in IR Reflection Absorption Spectroscopy (IRRAS) techniques [1-3]. Starting from work of Hartstein et al. [10] a new modification of IRRAS method was proposed, namely by using a combination of IR ATR technique with a deposition of metallic underlayer (on the top of IRE) or overlayer on the top of the analyte in order to achieve surface enhancement of IR signal similar with Surface Enhanced Raman Spectroscopy (SERS). Originally this technique was developed for polymer layer (or solid cast layer) investigation, therefore there was no problem to deposit a metallic layer on top of the ATR element or on top of the polymer layer $[1,2]$. As for the metal layer, firstly gold and silver (rarer copper) were used for the deposition similar to the initial developments of SERS technique. That is mainly due to a superior surface enhancement factor for $\mathrm{Au}$ and $\mathrm{Ag}$ [11]. However, later it was shown that some non-typical SERS metals (like Pt, Pd, Rh, Ni, Fe [1]) also demonstrate quite significant enhancement of the infrared signal. In analogy with SERS technique this new method was named as a SEIRA (surface enhanced infrared absorption).

The principles behind SEIRA $[12,13]$ can be found elsewhere and will not be discussed here in details. A clear explanation for the mechanism of the absorption enhancement remains elusive but the most prominent theories include the electromagnetic [14] and chemisorption [15] theories. In reality it is probable that both mechanisms contribute to the absorption enhancement. It is also recognised that the morphology of the metal films affects the enhancement factor [16]. Similar approaches utilising SEIRA and ATR have been used to analyse biological fluids $[17,18]$, environmental pollutants [19], organic semiconductor behaviour [20], as well as protein structures and interactions [21]. Therefore, there are numerous applications for this versatile method in many fields of science, medicine and engineering.

The main aim of this work is to develop a modified IR-ATR spectroscopy approach and to demonstrate the feasibility of the use of this technique for the qualitative analysis of biological molecules in solutions (i.e. in the liquid form). Therefore, in this report we demonstrate a modified SEIRA approach to enhance the infrared signal based on experimental investigations of selected biological specimens (namely Bovine Serum Albumin (BSA), Creatine, Salmon and Herring DNA) with this new setup. In the proposed methodology we use two new approaches. First of all, we avoid deposition of the metal neither onto the ATR (or IRE) element nor onto analyte. Instead we utilise metal coated glass substrate which was used as a top window placed on the top of the liquid sample deposited onto Ge hemispherical IRE (see Ref. [22] for details). We used $\mathrm{Al}$ as a metal for deposition onto thick $(\sim 4 \mathrm{~mm})$ glass substrate or in some cases onto widely available microscope glass slices (of $\sim 1 \mathrm{~mm}$ thick). We believe that Al layer provides sufficient surface enhancement, is easy to deposit and is easy attainable. The low cost and simple experimental setup make our method very attractive for a prospective commercialization and a widespread use.

\section{Experimental: Sample Preparations}

We analyzed a number of biological samples, such as various DNA, Glucose, Bilirubin, Creatine, etc. These substances are found in a various body fluid such as blood and urine. Monitoring of the concentrations of these substances is vital to the treatment and diagnostics of many pathological conditions such as cancer [23], liver cirrhosis, hepatitis [24], diabetes, renal disease [25], etc. However, in this report we included only four samples from the above list due to space limitations for a Short Communication paper. Salmon DNA, Herring DNA, Creatine and BSA and all other chemicals and reagents were purchased from Sigma-Aldrich in a powder form. To demonstrate the possibility of new technique these compounds were dissolved in Millipore water with concentration of $50 \%(\mathrm{w} / \mathrm{v}$ ) for all studied samples. In addition, all of the analytes were initially immobilized in a $\mathrm{KBr}$ tablet and FTIR absorbance spectra were measured. These were taken as a comparison to the surface enhanced absorption spectra. The concentrations of the analytes in $\mathrm{KBr}$ are as follows: Salmon DNA 5\% (w/w), Herring DNA 5\% (w/w), Creatine 1\% (w/w) and BSA 5\% (w/w).

\section{FTIR Measurements}

Infrared absorption spectra were measured on an FTS 6000 Fourier Transform Infrared (FTIR) spectrometer using Grazing Angle Attenuated Total Reflection (GATR) attachment from the Harrick Scientific Corporation with Ge hemispherical Internal Reflection Element (IRE). Absorption measurements were made on a thin layer of liquid (analyte) as shown in set up in Figure 1a (see also [22] for details). A thin film of analyte was obtained by liquid compression between the Ge ATR prism and a $\sim 4 \mathrm{~mm}$ thick Al coated glass substrate (see Figure 1a). In some cases, thick $(\sim 1 \mathrm{~mm}) \mathrm{mi}-$ croscope glass slide was used for Al layer deposition. The strength of window compression was changed using the GATR pressure applicator control. Measurements were performed in p-polarized light at a $60^{\circ}$ angle of incidence. The Ge IRE was carefully cleaned each time before a drop of liquid was placed on it. The glass substrate with Al layer was freshly prepared; new Al coated element was used for each experiment. A small drop of liquid was placed on the top of the ATR Ge prism, then the Al coated glass plate was placed on the top of the drop and the experiment was immediately run as the compression of the top glass window was increased. As a result of this the thickness of the analyte layer was decreased while the enhancement of SEIRA measurements increased.

The coating of thin Al layer (of $~ 0.1 \mu \mathrm{m}$ thick) was applied by evaporation of $\mathrm{Al}$ wire in a bell jar thermal evaporator (Edwards, Model E306A). The background pressure in the evaporator at the time of deposition was at least $6 \times 10^{-6}$ torr. Under these evaporation 
conditions, the $\mathrm{Al}$ film consists of pores, with diameters ranging from a few microns to tens of nanometers. The results of the surface porous structure of the substrates used for these experiments. analysis of $\mathrm{Al}$ coated glass substrate using atomic-force microscope (AFM) is shown in Figure 1b, which confirm the existence of the
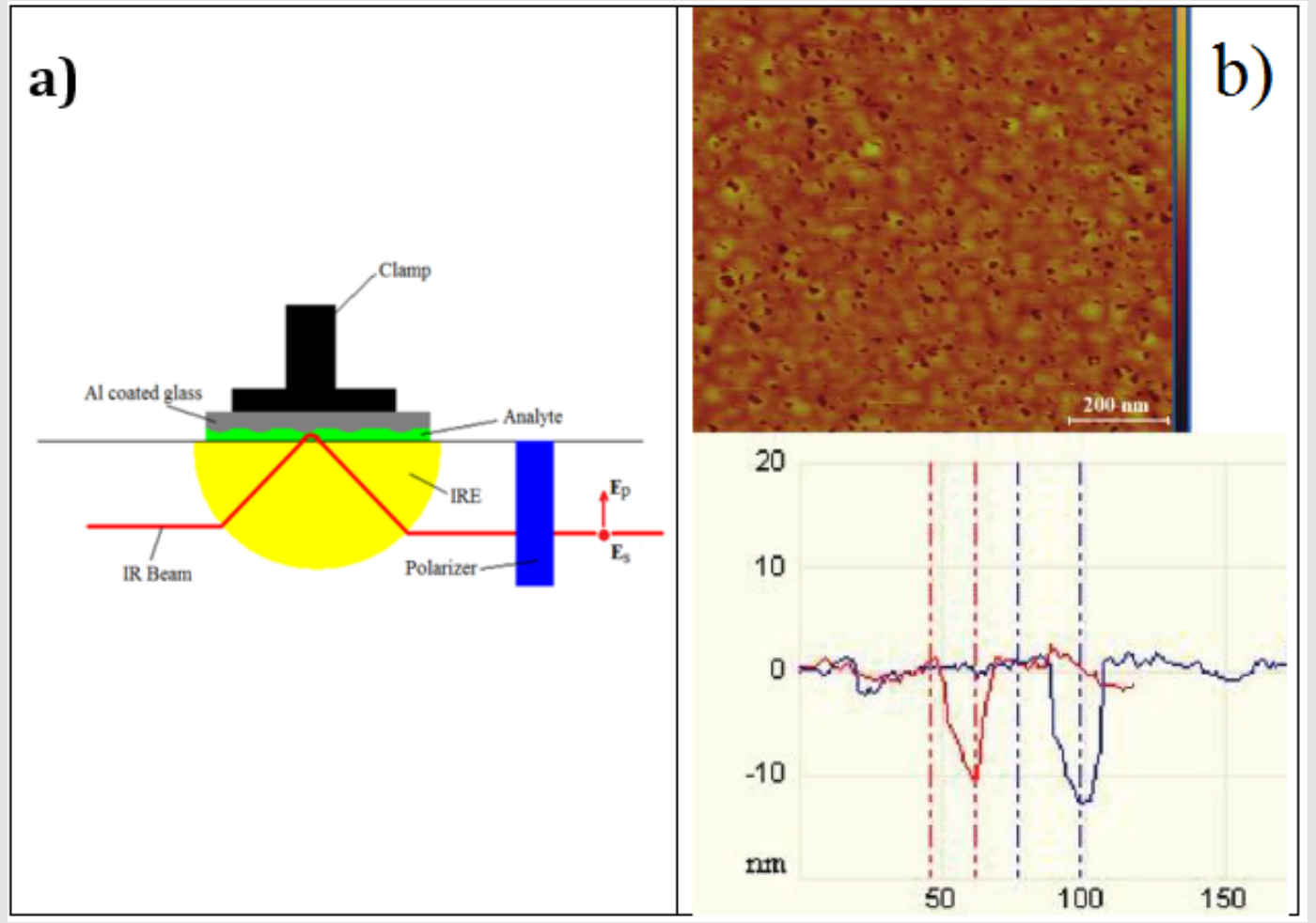

Figure 1:

a) Schematic of GATR attachment with $\mathrm{Al}$ coated top glass window for SEIRA measurements. The pressure applicator labelled as a Clamp.

b) AFM image of the Al-coated glass substrate shown from the top view (top picture) and for the depth profile (bottom graph).

\section{Results and Discussion}

The absorption spectra obtained for four biological samples, BSA, Salmon DNA, Herring DNA and Creatine are shown in Figures $2 \mathrm{a}-2 \mathrm{~d}$ respectively. In order to demonstrate the effect of infrared signal enhancement qualitatively we recorded infrared absorption spectra of investigated samples using 3 types of FTIR measurements: I - drop of liquid sitting free in the middle of $\mathrm{Ge}$ IRE element without top Al coated substrate; II - with top Al coated glass substrate placed under pressure on top of liquid drop; III analyte in powder form immobilised in a KBr tablet. For simplicity of presentation in this short report we compare only spectra registered with GATR attachment in p-polarised IR light, i.e. with and without top Al coated substrate. Infrared spectra of BSA, obtained using measurements I and II, are compared in Figure 2a. As can be seen from this figure spectrum of BSA, using top Al coated glass substrate, is significantly enhanced and demonstrates typical Amide I and Amide II bands at 1655 and $1543 \mathrm{~cm}^{-1}$, respectively. In addition to that, other vibrational bands are observed at 1396, $1339,1303,1242,903$ and $808 \mathrm{~cm}^{-1}$ in good correspondence with known IR bands of BSA from literature [26-28]. We note that the observed in this case a derivative-like shape of the enhanced IR spectrum is quite often seen in SEIRA spectra (see for example Refs. $[1,29,30]$ ). This is due to a dispersive effect which resulted in some molecular vibrations show up as negative absorption peaks, namely as reflectance maxima [1,29,31]. These spectral features can be simulated with Electromagnetic (EM) calculations based on Bergman model of Effective-Medium Approximation (EMA) [1,29].

The spectra registered for Salmon DNA (type of B-DNA) are shown in Figure 2b. In this case the IR spectrum appeared with all bands in absorption maxima and a significant enhancement (for some bands reached nearly of three orders of magnitude) is also obtained. The peak positions marked in Figure $2 \mathrm{~b}$ agree with previously measured IR spectra in Refs [32-34]. We should mention here that some IR bands registered for Salmon DNA in KBr tablets confirm the results obtained by SEIRA. This was also true to other investigated samples. Obviously, we cannot expect the total identity of all vibrational bands (in peak position and shape), bearing in mind a different effect of intermolecular interactions in various matrices and different polarisation conditions in these two experiments. The results for Herring DNA and for Creatine are shown in Figure 2c and Figure $2 \mathrm{~d}$, which also demonstrate quite substantial enhancement of vibrational bands, obtained with suggested in this work set up 
and Al metal layer for enhancement. Some observed IR bands are also supported by spectra registered earlier for these biological samples (see, for example, Refs. [35,36]). Thus, a modified SEIRA approach was demonstrated here, which can be easily utilised for measurements of liquid samples. In addition, the possibility of using Al as typical "non-SERS" metal for enhancement of IR signal was demonstrated with enhancement factor varied from 10 to 1000 for various IR bands. This method is easily accessible and does not require deposition of metal on expensive internal reflection element (which is in particularly important for the case of GATR attachment). Instead metal can be deposited on cheap glass substrates (or, if necessary, on some semiconductor window) which is used in this set up as top window during SEIRA measurements.
Finally, the thickness of the analyte can be control by the position of the pressure applicator. As a preliminary interpretation we would like to note that few mechanisms can be responsible for the IR absorption enhancement in these experiments. This can be due to electromagnetic enhancement as a result of the local field effect, due to multiple reflection of the incident light between two elements with high refractive index and finally due to adsorbance of the investigated analyte's molecules to the porous Al film structure. During our experiments we noticed that the enhancement of IR absorption (as well as relative intensity of different IR bands) depends on morphology of deposited $\mathrm{Al}$ film as well as on thickness of the liquid analyte. Obviously when film becomes thinner and thinner, most of the substance will be left inside the $\mathrm{Al}$ pores and chemisorbance effect will prevail over other mechanisms of the enhancement.

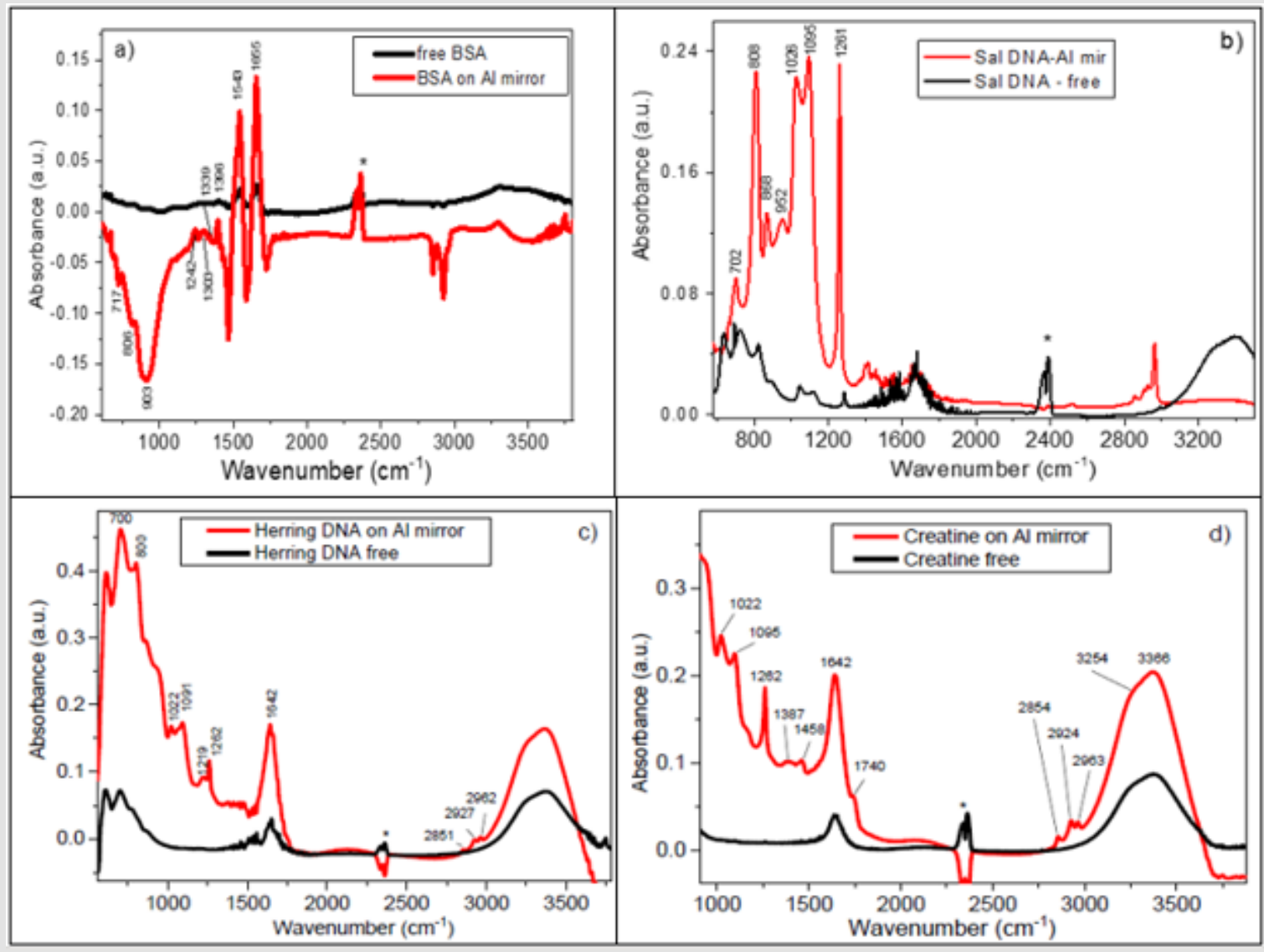

Figure 2: FTIR spectra registered with p-polarised IR light with GATR attachment for
a) BSA,
b) Salmon DNA,
c) Herring DNA and
d) Creatine

Note: The black lines correspond to the measurements taken from free liquid drop deposited onto Ge IRE, while red lines correspond to the case of liquid confined between Ge IRE and Al coated glass substrate. The double band in the region 2300$2400 \mathrm{~cm}^{-1}$ labelled with asterisk belongs to uncompensated $\mathrm{CO}_{2}$ from the air.

It is clear that more experimental and theoretical investigations using this approach are necessary in order to establish a complete quantitative analysis of biological samples (as well as any liquid sample) with weak IR bands. This short report presents only demonstration of the effect and a modified approach to SEIRA technique using grazing angle ATR technique and top substrate 
(made from dielectric or semiconducting material) coated by thin metal film. To conclude, we have developed a novel cheap and easy to use SEIRA method utilizing surface enhancement for the potential measurement of biochemical samples. This method overcomes some of the drawbacks of IR spectroscopy and provides an amplification of the analyte bands which allowed for detection of amido bands in the bulk water bands. However, further detailed studies will be necessary to understand mechanisms involved and optimize this technique. We believe that this method might find a range of important applications including analysis of very diluted biochemical and biomedical samples that will be of great value for medical diagnostics and forensic sciences.

\section{References}

1. Osawa M (2001) Surface-Enhanced Infrared Absorption. Topics Appl Phys 81: 163-187.

2. Griffiths P (2013) Surface-enhanced infrared absorption spectroscopy: Principles and applications. Spectrocs Prop Inorg Organomet Compd 44: 95-122.

3. Mun J, Lee D, So S, Badloe T, Rho J (2018) Surface-enhanced spectroscopy: Toward practical analysis probe. Applied Spectroscopy Reviews 54(1): $1-34$.

4. Ataka K, Stripp ST, Heberle J (2013) Surface-enhanced absorption spectroscopy (SEIRAS) to probe monolayer of membrane proteins. Biochim Biophys Acta 1828(10): 2283-2293.

5. Ataka K, Heberle J (2007) Biochemical applications of surface-enhanced infraredabsorption spectroscopy. Anal Bioanal Chem 388(1): 47-54.

6. Kazarian SG, Chan KLA (2006) Applications of ATR-FTIR spectroscopic imaging to biomedical samples. Biochim Biophys Acta 1758(7): 858867.

7. Shaw RA, Low Ying S, Leroux M, Mantsch HH (2000) Toward reagentfree clinical analysis: Quantitation of urine urea, creatinine, and total protein from the mid-infrared spectra of dried urine films. Clinical Chem 46(9): 1493-1495.

8. Hazen KH, Amold MA, Small GW (1998) Measurement of glucose and other anylates in undiluted human serum with near-infrared transmissions spectroscopy. Anal Chim Acta 371(2-3): 255-267.

9. Shaw RA, Kotowich S, Leroux M, Mantsch HH (1998) Multianalyte serum analysis using mid-infrared spectroscopy. Ann Clin Biochem 35: 624632.

10. Hartstein A, Kirtley JR, Tsang JC (1980) Enhancement of the Infrared Absorption from Molecular Monolayers with Thin Metal Overlayer. Phys Rev let 45: 201-204.

11. Zeman EJ, Schatz GC (1987) An Accurate Electromagnetic Theory Study of Surface Enhancement Factors for $\mathrm{Ag}, \mathrm{Au}, \mathrm{Cu}, \mathrm{Li}, \mathrm{Na}, \mathrm{AI}, \mathrm{Ga}, \mathrm{In}, \mathrm{Zn}$, and Cd. J Phys Chem 91(3): 634-643.

12. Fahrenfort J (1961) Attenuated total reflection: A new principle for the production of useful infrared spectra of organic compounds. Spectrochim Acta 17: 689.

13. Harrick NJ (1963) Total internal reflection and its application to surface studies. Ann NY Acad Sci 101(3): 928-959.

14. Osawa M, Ataka K (1992) Electromagnetic mechanism of enhanced infrared absorption of molecules adsorbed on metal island films. Surf Sci Lett 262(3): L118-L122.

15. Ishida KP, Griffiths PR (1994) Theoretical and Experimental Investigation of Internal Reflection at Thin Copper Films Exposed to Aqueous Solutions. Anal Chem 66(4): 522-530.

16. Jensen TR, Van Duyne RP, Johnson SA, Maroni VA (2000) Surface-
Enhanced Infrared Spectroscopy: A Comparison of Metal Island Films with Discrete and Nondiscrete Surface Plasmons. Applied spectroscopy 54(3): 371-377.

17. Weiling L, Kexin X, Qilian Y, Sixiang Z, Duogang R (2005) Determination of Multiple Components in Urine Using FT-MIR, NIR and FT-Raman Spectroscopic Technique. Proceed SPIE 5640: 610-615.

18. Mendelson Y, Clermont AC, Peura RA, Lin BC (1990) Blood Glucose Measurement by Multiple Attenuated Total Reflection and Infrared Absorption Spectroscopy. IEEE Trans Biomed Eng 37(5): 458-465.

19. McGuire MM, Carlson DL, Vikesland PJ, Kohn T, Grenier AC, et al. (2003) Applications of surface analysis in the environmental sciences: dehalogenation of chlorocarbons with zero-valent iron and ironcontaining mineral surfaces. Anal Chim Acta 496(1-2): 301-313.

20. Chua LL, Zaumseil J, Chang JF, Ou EC W, Ho PK H, et al. (2005) General observation of n-type field-effect behaviour in organic semiconductors. Nature 434: 194-199.

21. Rich PR, Iwaki M (2007) Methods to probe protein transitions with ATR infrared spectroscopy. Mol Bio Syst 3(6): 398-407.

22. Shaganov I, Perova T, Melnikov V, Dyakov S, Berwick K (2010) The Size Effect on the Infrared Spectra of Condensed Media under Conditions of 1D, 2D and 3D Dielectric Confinement. J Phys Chem C 114: 1607116081.

23. Zhong XY, Ladewig A, Schmid S, Wight E, Hahn S, et al. (2007) Elevated level of cell-free plasma DNA is associated with breast Cancer. Arch Gynecol Obstet 276(4): 327-331

24. Khan MH, Farrell GC, Byth K, Lyn R, Weltman M, et al. (2003) Which Patients With Hepatitis C Develop Liver Complications? Hepatology 31: 513-520.

25. Bellomo R, Ronco C, Kellum JA, Mehta RL, Palevsky P, et al. (2004) Acute renal failure-definition, outcome measures, animal models, fluid therapy and information technology needs: the Second International Consensus Conference of the Acute Dialysis Quality Initiative (ADQI) Group. Critical Care 8(4): R204-R212.

26. Grdadolnik J, Marechal Y (2001) Bovine Serum Albumin Observed by Infrared Spectrometry. I. Methodology, Structural Investigation, and Water Uptake. Biopolymers 62(1): 40-53.

27. Militello V, Casarino C, Emanuele A, Giostra A, Pullara F, et al. (2004) Aggregation kinetics of bovine serum albumin studied by FTIR spectroscopy and light scattering. Biophys Chem 107(2): 175-187.

28. Alhazmi HA (2019) FT-IR Spectroscopy for the Identification of Binding Sites and Measurements of the Binding Interactions of Important Metal Ions with Bovine Serum Albumin. Sci Pharm 87(5): 1-13.

29. Bjerke AE, Griffiths PR, Theiss W (1999) Surface-enhanced infrared absorption of CO on platinized platinum. Annal Chem 71: 1967-1974.

30. Krauth O, Fahsold G, Pucci A (1999) Asymmetric line shapes and surface enhanced infrared absorption spectrometry of $\mathrm{CO}$ adsorbed on thin $\mathrm{Fe}$ films on MgO(001). J Chem Phys 110: 3113-3117.

31. Lu GO, Sun SG, Cai LR, Chen SP, Tian ZW, et al. (2000) In situ FTIR spectroscopic studies of adsorption of $\mathrm{CO}, \mathrm{SCN}-$, and poly(ophenylenediamine) on electrodes of nanometer thin films of $\mathrm{Pt}, \mathrm{Pd}$, and $\mathrm{Rh}$ : Abnormal infrared effects (AIREs). Langmuir 16(2): 778-786.

32. Pichler A, Rüdisser S, Mitterböck M, Huber CG, Winger RH, et al. (1999) Unexpected BII Conformer Substate Population in Unoriented Hydrated Films of the d(CGCGAATTCGCG)2 Dodecamer and of Native B-DNA from Salmon Testes. Biophysical Journal 77(1): 398-409.

33. Rüdisser S, Pichler A, Winger RH, Liedl KR, Hallbrucker $\mathrm{A}$, et al. (2000) B-DNA's dynamics and conformational substates revealed by calorimetric enthalpy relaxation and Fourier transform infrared spectroscopy. J Molec Liquids 86(1-3): 137-149.

34. Taillandier A, Liquier J (1992) Infrared Spectroscopy of DNA. Methods Enzymol 211: 307-315. 
35. Globus TR, Woolard DL, Samuels AC, Gelmont BL, Hesler J, et al. (2002) Submillimeter-wave Fourier transform spectroscopy of biological macromolecules. J Appl Phys 91: 6105-6113.

\section{ISSN: 2574-1241}

DOI: $10.26717 /$ BJSTR.2020.24.004049

Tatiana S Perova. Biomed J Sci \& Tech Res

(C) (P) This work is licensed under Creative

Submission Link: https://biomedres.us/submit-manuscript.php
36. Kutorasinska J, Zetkowitz Z, Janeczko K, Sandt C, Dumas P, et al. (2013) Differences in the hippocampal frequency of creatine inclusions between the acute and latent phases of pilocarpine model defined using

$\begin{array}{ll}\text { BIOMEDICAL } & \text { Assets of Publishing with us } \\ \text { RESEARCHES } & \text { - Global archiving of articles } \\ & \text { - Immediate, unrestricted online access } \\ & \text { - Rigorous Peer Review Process } \\ & \text { - Authors Retain Copyrights }\end{array}$

